\title{
Subgraphs and network motifs in geometric networks
}

\author{
Shalev Itzkovitz, Uri Alon \\ Departments of Molecular Cell Biology and Physics of Complex Systems, \\ Weizmann Institute of Science, Rehovot, Israel 76100
}

\begin{abstract}
Many real-world networks describe systems in which interactions decay with the distance between nodes. Examples include systems constrained in real space such as transportation and communication networks, as well as systems constrained in abstract spaces such as multivariate biological or economic datasets and models of social networks. These networks often display network motifs: subgraphs that recur in the network much more often than in randomized networks. To understand the origin of the network motifs in these networks, it is important to study the subgraphs and network motifs that arise solely from geometric constraints. To address this, we analyze geometric network models, in which nodes are arranged on a lattice and edges are formed with a probability that decays with the distance between nodes. We present analytical solutions for the numbers of all 3 and 4-node subgraphs, in both directed and non-directed geometric networks. We also analyze geometric networks with arbitrary degree sequences, and models with a field that biases for directed edges in one direction. Scaling rules for scaling of subgraph numbers with system size, lattice dimension and interaction range are given. Several invariant measures are found, such as the ratio of feedback and feed-forward loops, which do not depend on system size, dimension or connectivity function. We find that network motifs in many real-world networks, including social networks and neuronal networks, are not captured solely by these geometric models. This is in line with recent evidence that biological network motifs were selected as basic circuit elements with defined information-processing functions.
\end{abstract}

PACS numbers: $05,89.75$

\section{INTRODUCTION}

Many systems in nature can be represented as complex networks [1, 2, 3, 4]. Often, natural and engineered networks have an underlying geometric arrangement. In such networks, nodes are embedded in a geometric space, and edges tend to link nodes that are close neighbors. Examples include the physical layout of the internet 5, 6, 7, 8], transportation networks [9, 10], power grids 4, 8], as well as networks of wiring between neurons [11, 12, 13, 14] or cortical areas [15, 16].

A geometric constraint does not have to be of spatial origin. In models of social [17] or world-wide-web networks [18, 19], nodes may be assigned attributes (e.g. language and field of interest of web pages, occupation and residence in social networks) and links may be correlated with closeness in this attribute-space [20, 21]. Geometric networks can also arise from analysis of multivariate datasets. For example, networks have been proposed to describe gene expression [22] or economic datasets 23], where distance between nodes corresponds to high correlation coefficient in the dataset. These networks can be embedded in a high dimensional Euclidean vector space.

Many of these diverse networks have been recently found to display network motifs $[8,14,24,25]$ : a characteristic set of recurring subgraphs which occur much more often than in randomized networks with the same degree sequence. Different networks usually display different motifs, and motifs can be used to characterize families of networks. In biological networks, it has been demonstrated that each of the motifs can carry out a key infor- mation processing function [26, 27, 28, 29, 30, 31, 32.

It is of interest to study the origin of network motifs in each real-world network. In particular, it is of interest to compare the network local structure to that of model networks which have a similar geometric constraint. For example, the researchers who mapped the synaptic wiring of $C$. elegans speculated that :"The abundance of triangular connections in the nervous system of C. elegans may thus simply be a consequence of the high levels of connectivity that are present within neighbourhoods" 11]. An analysis of the abundance of subgraphs in purely geometric networks, can help discern whether the motifs arise based on simple geometry, or whether they arise due to additional optimization or design of the network [33, 34].

To address this, we study geometric network models, in which nodes are arranged on a lattice, and edges are placed randomly between nodes with a probability $F(r)$ that decays with the distance between nodes. Several features of related models were previously studied [35, 36, 37, 38, 39]. These features include degree distributions [35, 36], diameters [37] and clustering coefficients [38]. Here we focus on the subgraph content of these networks. We consider non-directed and directed networks, as well as cases where directed edges are biased in a particular spatial direction. We present an analytical solution for the numbers of small subgraphs and the scaling of all types of subgraphs with system size and lattice dimensionality. We find invariants that can be used to easily compare networks with geometric models. 
(a)
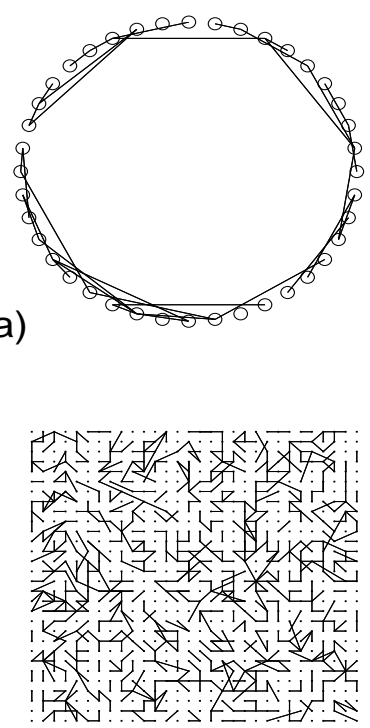

(c)
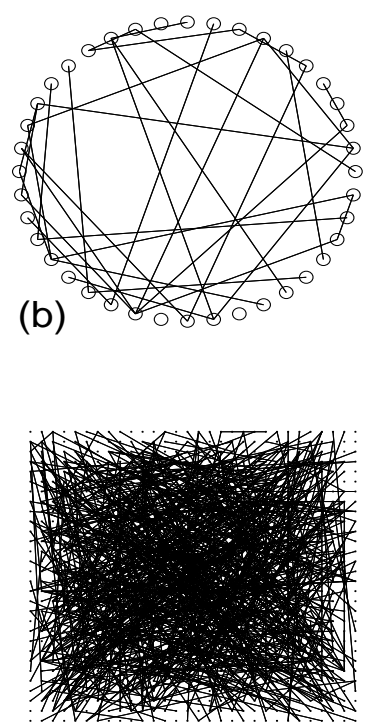

(d)

FIG. 1: Non-directed geometric networks and their randomized versions. (a) $1 \mathrm{~d}$ geometric network with $N=40, R=7,\langle k\rangle=2$. (b) $1 \mathrm{~d}$ randomized version. (c) 2 d geometric network with $N=900, R=1,\langle k\rangle=2$. (d) 2 d randomized version. The 2 d network shown has non-continuous boundary conditions, for clarity.

\section{RESULTS}

\section{A. Non-directed geometric model}

In the geometric model, $N$ nodes are arranged in a $d$-dimensional Euclidean lattice with toroidal (continuous) boundary conditions (Fig 1). Non-directed edges are placed at random according to a connectivity density function $F(x, y)$ (where $F(x, y)=F(r(x, y)$ ), and $r$ is the distance between nodes $x$ and $y)$. For each pair of nodes, $x$ and $y$, a random number $p$ is generated, and an edge is placed if $p<F(x, y) . F(r)$ is a decaying function with a range $R=\int r F(r) \overrightarrow{d r}$. We consider the case where $R$ is much larger than the lattice spacing $(R>>1)$ but where the effective connectivity neighborhood of each node is much smaller than the system size $R^{d}<<N$. In this case, the mean number of edges per node is

$$
\langle k\rangle=\int F(r) \overrightarrow{d r}
$$

The degree distribution, the distribution of number of edges per node, $P(k)$ is Poissonian, with a mean of $\langle k\rangle$ (assuming that $F(r)$ decays sufficiently rapidly [65]). Therefore, a random network ensemble that preserves the degree distribution of the geometric network, is the Erdős-Rényi model [40, 41, 42, 43] with $N$ nodes where edges are placed at random with probability $p_{E r d}=$ $\langle k\rangle / N$.

We now calculate the mean number of appearances of a given subgraph in the geometric model. The probability for the subgraph may be expressed in terms of overlap integrals of the function $F(r)$. For example, the triangle subgraph tends to occur when three nodes, $x, y$ and $z$ are sufficiently close, as expressed by the integral

$$
\left\langle G_{\Delta}\right\rangle=\frac{N}{6} \iint F(0, y), F(0, z), F(y, z) \overrightarrow{d y} \overrightarrow{d z}
$$

Where without loss of generality $x$ is at the origin. The factor $1 / 6$ is due to the symmetry, where the same triangle can be counted if $y$ or $z$ serve as the origin, and if $y$ and $z$ are interchanged when $x$ is the origin. The symmetry factor can be calculated based on the symmetry of each subgraph (one over the number of permutations of nodes that lead to an isomorphic subgraph).

In table I, we present the number of appearances of all three and four-node subgraphs in a non-directed geometric network. The results in the table apply to the case of sparse networks, where $\langle k\rangle<<R^{d}$ (as occurs in almost all real world networks). The results are for two connectivity functions (Fig 2). The first is a Gaussian connectivity function, where

$$
F_{g}(r)=k\left(2 \pi R^{2}\right)^{-d / 2} \exp \left(-r^{2} / 2 R^{2}\right)
$$

and where

$$
r^{2}=\sum_{i}\left(x_{i}-y_{i}\right)^{2}
$$

is the $L^{2}$ norm. $\left\{x_{i}\right\},\left\{y_{i}\right\}$ denote the d-dimensional coordinates of nodes $x$ and $y$. The second connectivity function is a hard-cube connectivity function :

$$
F_{c}(r)=k(2 R)^{-d} \Theta(r<R)
$$




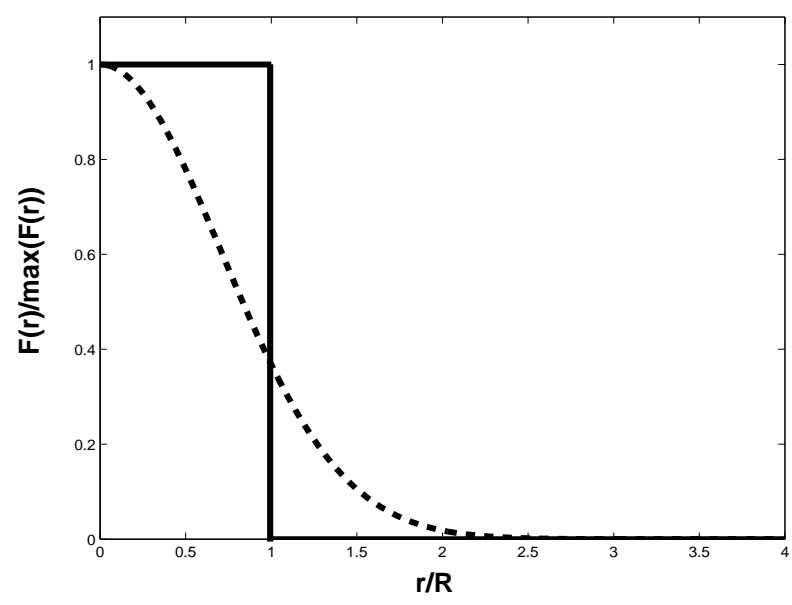

FIG. 2: Gaussian (dashed) and hard-cube (bold) connectivity functions.

where

$$
r=\max \left|\left(x_{i}-y_{i}\right)\right|
$$

is the $L^{\infty}$ norm, and $\Theta$ is a step function. Similar overlap integrals (Appendix A) appear in the calculation of virial coefficients [57], and in calculations of percolation thresholds [58].

\section{B. Scaling of subgraph numbers with system size and dimension}

We present a simple scaling argument for the subgraph content of geometric networks. In this picture, the neighborhood of each node at distances smaller than $R$ is similar to an Erdős-Rényi network. The number of appearances of a subgraph with n nodes and $g$ edges in an ErdossRényi network of size $N_{E}$ and mean connectivity $\langle k\rangle$ has been shown to scale as [43, 44]

$$
\left\langle G_{E r d}\right\rangle \sim N_{E}^{n-g}\langle k\rangle^{g}
$$

In total, there are on the order of $N / R^{d}$ such Erdös-like domains in the entire network, each one of size $N_{E}=R^{d}$. Therefore, the scaling of the number of appearances of subgraph $G$ in the geometric network is:

$$
\left\langle G_{\text {geom }}\right\rangle \sim\left(N / R^{d}\right) N_{E}^{n-g}\langle k\rangle^{g}
$$

which results in :

$$
\left\langle G_{\text {geom }}\right\rangle \sim N R^{(n-g-1) d}\langle k\rangle^{g}
$$

All subgraphs in the geometric network scale with network size $N$ as $G_{\text {geom }} \sim N$ (Table I). This is in contrast to Erdős networks, in which $G \sim N^{n-g}$. Therefore, in the limit of large system size, all subgraphs in which $g \geq n$ will be network motifs. This includes triangles, squares, and aggregates of triangles.

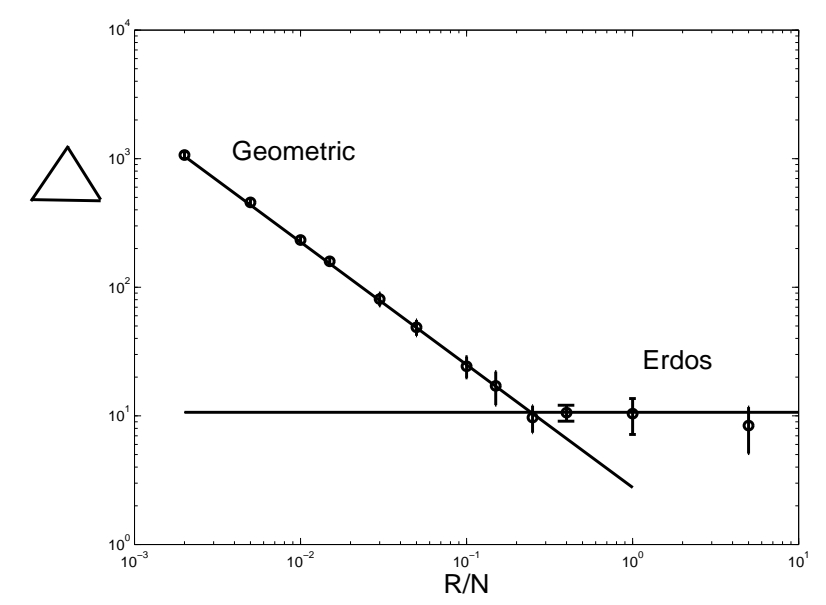

FIG. 3: Scaling regimes of the geometric model $(N=$ $5000, d=1, k=4)$. At low $R / N$ ratios triangles scale as $(R / N)^{-1}$. At high $R / N$ ratios the network becomes an Erdős network and triangles scale as $(R / N)^{0}$.

The present scaling argument also provides the regime in which finite-size effects begin to be important. Finite size effects begin when $R^{d} \sim N$. When $R^{d}>N$, the entire network essentially behaves as an Erdös netwok, and the scaling crosses over to Erods-Rényi scaling (Fig 3 , see also [38]).

The scaling relations can also be written in terms of the network clustering coefficient [1, 2, 3, 4, 45, 46, 47], which is related to the ratio of triangles to $\mathrm{V}$-shaped subgraphs (Table I):

$$
C=3 \frac{G_{2}}{G_{1}} \sim \frac{\langle k\rangle}{R^{d}}
$$

Inserting this into Eq. (9) we get:

$$
\left\langle G_{\text {geom }}(n, g)\right\rangle \sim N C^{g-n+1}\langle k\rangle^{d(n-1)-g(d-1)}
$$

In geometric networks, knowing $C$ and $\langle k\rangle$ is sufficient to find the scaling of all subgraphs. This is not the case for other types of networks: In general, in non-geometric networks $C$ does not determine the number of four-node patterns (e.g. diamonds - pattern 6 in Table I) and larger motifs.

\section{Directed isotropic geometric model}

We now consider the case where each edge has a direction, yielding a directed network. This network is built in the same way as the non-directed network, except that each pair of nodes $x, y$ is considered twice, and directed edges can connect $x$ to $y$ and $y$ to $x$. The mean number of outgoing edges per node is equal to the mean number of incoming edges:

$$
\left\langle k_{\text {in }}\right\rangle=\left\langle k_{\text {out }}\right\rangle=\int F(r) \overrightarrow{d r}
$$




\begin{tabular}{|c|c|c|c|c|c|c|c|c|c|}
\hline subgraph & pattern & nodes & edges & geometric model & $1 \mathrm{~d} f_{G}$ & $2 \mathrm{~d} f_{G}$ & $1 \mathrm{~d} f_{h c}$ & $2 \mathrm{~d} f_{h c}$ & Erdős \\
\hline 1 & $\Lambda$ & 3 & 2 & $f_{1} N\langle k\rangle^{2}$ & $2^{-1}$ & $2^{-1}$ & $2^{-1}$ & $2^{-1}$ & $N\langle k\rangle^{2} / 2$ \\
\hline $2^{*}$ & $\triangle$ & 3 & 3 & $f_{2} N\langle k\rangle^{3} / R^{d}$ & $(6 \sqrt{6 \pi})^{-1}$ & $(36 \pi)^{-1}$ & $3 / 48$ & $3 / 128$ & $\langle k\rangle^{3} / 6$ \\
\hline 3 & $\bigwedge$ & 4 & 3 & $f_{3} N\langle k\rangle^{3}$ & $6^{-1}$ & $6^{-1}$ & $6^{-1}$ & $6^{-1}$ & $N\langle k\rangle^{3} / 6$ \\
\hline 4 & $\square$ & 4 & 3 & $f_{4} N\langle k\rangle^{3}$ & $2^{-1}$ & $2^{-1}$ & $2^{-1}$ & $2^{-1}$ & $N\langle k\rangle^{3} / 2$ \\
\hline $5^{*}$ & $\triangle$ & 4 & 4 & $f_{5} N\langle k\rangle^{4} / R^{d}$ & $(2 \sqrt{6 \pi})^{-1}$ & $(12 \pi)^{-1}$ & $3 / 16$ & $9 / 128$ & $\langle k\rangle^{4} / 2$ \\
\hline $6^{*}$ & $\square$ & 4 & 4 & $f_{6} N\langle k\rangle^{4} / R^{d}$ & $(16 \sqrt{2 \pi})^{-1}$ & $(64 \pi)^{-1}$ & $1 / 24$ & $1 / 72$ & $\langle k\rangle^{4} / 8$ \\
\hline $7^{*}$ & $\triangle$ & 4 & 5 & $f_{7} N\langle k\rangle^{5} / R^{2 d}$ & $\left(16 \sqrt{(2) \pi)^{-1}}\right.$ & $(64 \pi)^{-1}$ & $7 / 192$ & $49 / 9216$ & $\langle k\rangle^{5} / 4 N$ \\
\hline $8^{*}$ & $\bowtie$ & 4 & 6 & $f_{8} N\langle k\rangle^{6} / R^{3 d}$ & $\left(192 \sqrt{2} \pi^{3 / 2}\right)^{-1}$ & $\left(3072 \pi^{3}\right)^{-1}$ & $1 / 384$ & $1 / 6144$ & $\langle k\rangle^{6} / 24 N^{2}$ \\
\hline
\end{tabular}

TABLE I: Numbers of non-directed three and four-node subgraphs in the geometric model with $N$ nodes, mean connectivity $\langle k\rangle$, range $R$ and dimension $d$. Pre-factors, $f$, are for $1 d$ and $2 d$ Gaussian connectivity function $\left(f_{G}\right)$ and hard-cube connectivity function $\left(f_{h c}\right)$. Also shown are the mean number of subgraphs in Erdös networks with mean connectivity $\langle k\rangle$. Stars represent subgraphs that are network motifs in the limit of large system size.

\begin{tabular}{|c|c|c|c|c|c|c|c|c|}
\hline subgraph pattern & edges & geometric model & $1 \mathrm{~d} f_{G}$ & $2 \mathrm{~d} f_{G}$ & $1 \mathrm{~d} f_{h c}$ & $2 \mathrm{~d} f_{h c}$ & field factor & Erdős \\
\hline 1 & 2 & $f_{1} N\langle k\rangle^{2}$ & $2^{-1}$ & $2^{-1}$ & $2^{-1}$ & $2^{-1}$ & 1 & $N\langle k\rangle^{2} / 2$ \\
\hline 2 & 2 & $f_{2} N\langle k\rangle^{2}$ & 1 & 1 & 1 & 1 & 1 & $N\langle k\rangle^{2}$ \\
\hline 3 & 2 & $f_{3} N\langle k\rangle^{2}$ & $2^{-1}$ & $2^{-1}$ & $2^{-1}$ & $2^{-1}$ & 1 & $N\langle k\rangle^{2} / 2$ \\
\hline $4 *$ & 3 & $f_{4} N\langle k\rangle^{3} / R^{d}$ & $(2 \sqrt{\pi})^{-1}$ & $4 \pi^{-1}$ & $2^{-1}$ & $4^{-1}$ & $\mathrm{pq}$ & $\langle k\rangle^{3}$ \\
\hline $5 *$ & 3 & $f_{5} N\langle k\rangle^{3} / R^{d}$ & $(2 \sqrt{\pi})^{-1}$ & $4 \pi^{-1}$ & $2^{-1}$ & $4^{-1}$ & $\mathrm{pq}$ & $\langle k\rangle^{3}$ \\
\hline $6 *$ & 3 & $f_{6} N\langle k\rangle^{3} / R^{d}$ & $(3 \sqrt{6 \pi})^{-1}$ & $(18 \pi)^{-1}$ & $(3 / 8) / 3$ & $(3 / 8)^{2} / 3$ & $\mathrm{pq}$ & $\langle k\rangle^{3} / 3$ \\
\hline $7 *$ & 3 & $f_{7} N\langle k\rangle^{3} / R^{d}$ & $(\sqrt{6 \pi})^{-1}$ & $6 \pi^{-1}$ & $3 / 8$ & $(3 / 8)^{2}$ & $\left(p^{3}+4 p q+q^{3}\right) / 6$ & $\langle k\rangle^{3}$ \\
\hline $8 *$ & 4 & $f_{8} N\langle k\rangle^{4} / R^{2 d}$ & $(8 \pi)^{-1}$ & $\left(32 \pi^{2}\right)^{-1}$ & $(1 / 4) / 2$ & $(1 / 4)^{2} / 2$ & $p^{2} q^{2}$ & $\langle k\rangle^{4} / 2 N$ \\
\hline $9 *$ & 4 & $f_{9} N\langle k\rangle^{4} / R^{2 d}$ & $(4 \sqrt{5} \pi)^{-1}$ & $\left(40 \pi^{2}\right)^{-1}$ & $(3 / 16) / 2$ & $(3 / 16)^{2} / 2$ & $p q\left[a p q+b\left(p^{2}+q^{2}\right)\right]$ & $\langle k\rangle^{4} / 2 N$ \\
\hline $10 *$ & 4 & $f_{10} N\langle k\rangle^{4} / R^{2 d}$ & $(2 \sqrt{5} \pi)^{-1}$ & $\left(20 \pi^{2}\right)^{-1}$ & $3 / 16$ & $(3 / 16)^{2}$ & $p q\left[c p q+d\left(p^{2}+q^{2}\right)\right]$ & $\langle k\rangle^{4} / N$ \\
\hline $11 *$ & 4 & $f_{11} N\langle k\rangle^{4} / R^{2 d}$ & $(4 \sqrt{5} \pi)^{-1}$ & $\left(40 \pi^{2}\right)^{-1}$ & $(3 / 16) / 2$ & $(3 / 16)^{2} / 2$ & $p q\left[a p q+b\left(p^{2}+q^{2}\right)\right]$ & $\langle k\rangle^{4} / 2 N$ \\
\hline $12 *$ & 5 & $f_{12} N\langle k\rangle^{5} / R^{3 d}$ & $\left(8 \pi^{3 / 2}\right)^{-1}$ & $\left(64 \pi^{3}\right)^{-1}$ & $3 / 32$ & $(3 / 32)^{2}$ & $p^{2} q^{2}$ & $\langle k\rangle^{5} / N^{2}$ \\
\hline $13 *$ & 6 & $f_{13} N\langle k\rangle^{6} / R^{4 d}$ & $\left(48 \sqrt{3} \pi^{2}\right)^{-1}$ & $\left(1152 \pi^{4}\right)^{-1}$ & $(3 / 64) / 6$ & $(3 / 64)^{2} / 6$ & $p^{3} q^{3}$ & $\langle k\rangle^{6} / 6 N^{3}$ \\
\hline
\end{tabular}

TABLE II: Numbers of directed 3-node subgraphs in the geometric model with $N$ nodes, mean connectivity $\langle k\rangle$, range $R$ and dimension $d$. Subgraphs are grouped according to number of edges. Pre-factors, $f$, are for $1 d$ and $2 d$ Gaussian connectivity function $\left(f_{G}\right)$ and hard-cube connectivity function $\left(f_{h c}\right)$. Field factors are for the $1 d$ Gaussian model $(a=0.27, b=0.365, c=$ $0.73, d=0.135)$. Also shown are the mean number of subgraphs in Erdös networks with mean connectivity $\langle k\rangle$. Stars represent subgraphs that are network motifs in the limit of large system size. Note that subgraphs $4,5,8$ are not network motifs when compared to randomized networks that preserve the degree distribution of both single and mutual edges [8, 25, 44] 


\begin{tabular}{|c|c|c|c|c|c|c|}
\hline & social & WWW & neurons-all & neurons-strong & geometric $p=1$ & geometric $p \rightarrow 0$ \\
\hline & $1(1): 13$ & $1(620): 400$ & $1(72): 22$ & $0: 40$ & $1: 3$ & $0: 1$ \\
\hline
\end{tabular}

TABLE III: Subgraph ratios in real world networks and in the geometric model. Networks are (i) social network 1 of $[8], N=67$, $E=182$, (ii) WWW hyperlink network 3 of [8] (source [19]), $N=47978, E=235441$. (iii) neural synaptic connections in C. elegans, $N=280, E=2170[8,11]$. (iv) strong neural synaptic connections in C. elegans, $N=280, E=400$ [14] (only connections with 5 or more synapses). Brackets indicate the absolute count for the subgraph taken as 1 in the ratio. The ratios for the isotropic geometric model $(p=1)$ apply to the poissonian model and for any dimension and connectivity function. Ratios 3 and 4 in the isotropic model apply also to geometric networks with arbitrary degrees. The ratios for the fully biased anisotropic geometric model $(p=0)$ apply to the $1 \mathrm{~d}$ geometric model with gaussian connectivity function. In ratios 3,4 for the WWW network a sampling algorithm for subgraph counting was applied [56] with $10^{6}$ samples.

We find that the same scaling arguments hold. The subgraphs fall into classes according to the number of nodes and edges $n, g$. Table II shows the result for threenode subgraphs for $d=1$ and 2. The 3 -node subgraphs fall into five classes, corresponding to subgraphs with $g=2,3,4,5$ and 6 edges. In each family, the scaling is the same, but the prefactors generally differ and depend on the dimensionality and on the form of $F(r)$.

\section{Geometric networks with arbitrary degree sequences}

Real-world networks often have degree sequences which are quite different from Poissonian. For example, many networks have heavy-tailed degree distributions 1, 2, 3, 4, 48, 49, 50, 51, 52, 53, 54, 55].

A heavy-tailed degree distribution in random networks has been shown to strongly affect the counts of certain subgraphs 44]. In particular, certain subgraphs appear much more often in random networks with heavy-tailed degrees than in Erdös networks. For example, subgraphs in networks with a scale-free out-degree $P(k) \sim k^{-\gamma}$ and compact in-degree have been shown to scale with network size $N$ as [44]:

$$
\langle G\rangle \sim N^{\alpha}
$$

where $\alpha$ is related to the number of subgraph nodes $n$, subgraph edges $g$ and maximal subgraph out-degree $s$ :

$$
\alpha=\left\{\begin{array}{cc}
n-g+s-1 & \gamma \leq 2 \\
n-g+s-\gamma+1 & 2<\gamma<s+1 \\
n-g & \gamma \geq s+1
\end{array}\right.
$$

The geometric models discussed above have a Poissonian degree distribution. An interesting extension of geometric models, which allows for heavy-tailed sequence has been recently studied by Havlin and colleagues. The scaling of path lengths in that model was derived [39].
Here, we consider subgraphs in a related, directed lattice model, with an arbitrary outgoing degree sequence $P(k)$. In this model, each node in the lattice is assigned an degree $k$ drawn from the distribution $P(k)$. $k$ outgoing edges are then randomly connected to other nodes according to the connectivity function $F(r)$. This results in a geometric model, with outgoing degree distribution $P(k)$, which can be heavy-tailed, and a compact incoming degree distribution. We note that several real-world directed networks have compact in-degree and heavy tailed out-degree, including biological [24, 25] and technological networks [54, 55].

We now derive scaling relations for the number of subgraphs in geometric networks with a heavy-tailed outgoing degree sequence:

$$
P(k) \sim k^{-\gamma}
$$

We consider the limit where the hubs in the network do not exceed the typical size of the neighborhood of each node, which scales as $R^{d}$. The mean hub size scales as $[39$, 44, 52, 53]:

$$
T \sim N^{1 /(\gamma-1)}
$$

Thus, we consider the case where $N^{1 /(\gamma-1)} \ll R^{d} \ll N$.

The network can be considered as a collection of $N / R^{d}$ subnetworks of size $N_{n}=R^{d}$, each with scaling according to Eq. 13. For the entire network, one finds that the number of subgraphs $\mathrm{G}$ scales as

$$
\langle G\rangle \sim\left(N / R^{d}\right) N_{n}^{\alpha}=N R^{(\alpha-1) d}
$$

with $\alpha$ given in Eq. 14. All subgraphs numbers scale as $N$, and have an $R$ dependance that depends on $\gamma$ and the subgraph topology. Subgraphs with large maximal out-degree $s$ tend to appear more often than subgraphs with smaller $s$. 


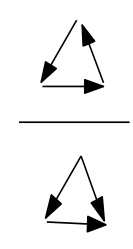

(a)

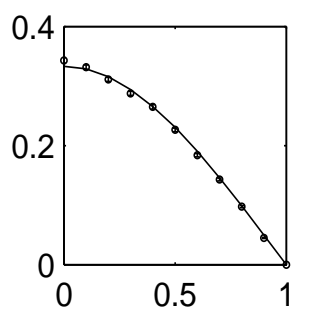

field strength (1-p)

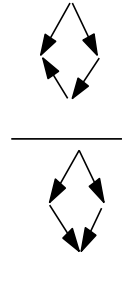

(b)

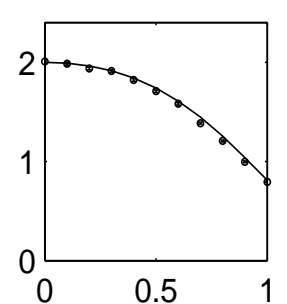

field strength $(1-p)$

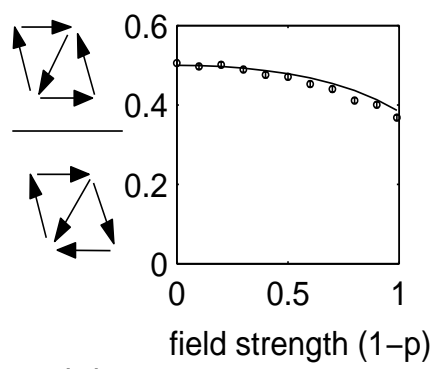

(c)

FIG. 4: Three subgraph ratios for different field strengths in an anisotropic geometric model with gaussian connectivity function. $d=1, N=2000, R=30,\langle k\rangle=8$. Shown are simulation results (a-c) and theoretical curves, obtained from Table II (a) and Appendix B (b,c). Numerical standard errors are smaller than the dot size.

\section{E. Invariant ratios}

It is of interest to find invariant measures which apply to geometric networks independent of the dimensionality $d$ and the form of $F(r)$. Several such invariants can be found. An example is the ratio of the numbers of two subgraphs, the feedback loop and the feed-forward loop (subgraphs 6 and 7 in Table II). The ratio of these two subgraphs is $1: 3$, regardless of $d$ and $F(r)$. Similarly, the ratio of subgraphs 9,10 and 11 is $1: 2: 1$. The ratios of subgraphs 1,2 and 3 is $1: 2: 1$. Table III shows these ratios (ratios 1 and 2) for several real world networks. The ratios in real world networks generally differ significantly from the ratios in isotropic geometric networks.

The above ratios are invariant in the poissonian geometric model, but can change if one considers geometric networks with an arbitrary degree sequence. For example, the feed-forward loop subgraph (subgraph 7 in Table II) has a node with 2 outgoing edges $(s=2)$, whereas the feedback loop (subgraph 6 in Table II) has $s=1$. This would result in an increased abundance of feed-forward loops for geometric networks with heavy-tailed degree sequence.

We therefore sought ratios of subgraphs which do not depend on the degree sequence. These are subgraphs which have the same subgraph degree sequences. The class of four-node directed subgraphs contains several examples of subgraphs with identical degree sequences. Two examples are shown in Table III (ratios 3 and 4). These ratios are invariant with respect to the degree sequence of the network model, as well as to the dimensionality $d$ and the form of $F(r)$

\section{F. Directed anisotropic geometric model}

In some systems there are preferred directions in which the probability of a directed connection is larger than in other directions. To model this, we consider a $d=1$ lattice, and introduce a bias that favors edges in one direction. In this case, a probability of a directed edge to the right is $F_{\text {right }}=p F(r)$ and an edge to the left is $F_{\text {left }}=q F(r)$, such that $p+q=2$ (the sum $=2$ is chosen to preserve the same $\langle k\rangle$ as for the isotropic model). The calculation is somewhat more intricate in this case, as the overlap integrals must be evaluated for different orderings of the nodes (Appendix B). The expression for each subgraph is multiplied by a 'field factor', which depends on the subgraph topology. The results for 3 -node directed subgraphs are shown in Table II.

The scaling is the same as in the isotropic case, except in the limit $p=0$ (or $q=0)$. In the latter limit, no cycles are allowed (the network is a directed acyclic graph). The only closed three-node pattern is the feed-forward loop (subgraph 7 in Table II).

The relative abundance of the various subgraphs depends on the 'field' $p$ (Table II). Subgraphs with mutual edges (edges in both direction between two nodes) are biased against since mutual edges always contain one edge that goes against the field. The ratios that were invariant in the isotropic case are no longer independent of $d$ and $F(r)$. Several of these ratios are plotted as a function of $p$ in Fig 4. Table III shows the ratios in real-world networks for the fully biased anisotropic geometric network.

\section{DISCUSSION}

This study presented analytical results for the subgraph content of geometric network models. We find scaling rules for the number of appearances of each subgraph as a function of network size and lattice dimensionality. The scaling is very different from Erdős-Rényi networks for most subgraphs. We find certain ratios of subgraph appearances in isotropic directed geometric networks, which are 'invariants' in the sense that they do not depend on dimension and connectivity function.

Geometric networks show distinct network motifs. All of the subgraphs scale as $N^{1}$, whereas they scale as $N^{n-g}$ 
in the corresponding random networks with the same degree sequence. Therefore all subgraphs with $g \geq n$ are network motifs in geometric networks. In most real-world networks studied so far, only a subset of these subgraphs are network motifs [8, 14, 24, 25], suggesting that additional constraints and optimization is at play, beyond isotropic geometric constraints.

The network motifs in real-world networks appear to be 'extensive variables', in the sense that their concentration $c=G / N$ does not decrease with $N$, but scales as $N^{0}$ 25], whereas their concentration in corresponding randomized networks decreases with $N$. A similar property is found for the concentration of motifs in geometric networks, which scales as $c=G / N \sim N^{0}$, and $c=G / N \sim N^{n-g-1}<N^{-1}$ in randomized networks.

Table III shows that the abundance of feedback loops relative to feed-forward loops in social and world-wideweb networks is much less than expected from an isotropic geometric constraint 66].

Social networks which represent positive sentiments between individuals in a group are known to be rich in transitive relations [8, 59, 60]: If $X$ 'likes' $Y$ and $Y$ likes $Z, X$ tends to also like $Z$. In these networks, intransitive triplets of nodes $(X \rightarrow Y, Y \rightarrow Z$, but $X \nrightarrow Z)$ are known to be relatively rare. Feedback loops might be rare because they contain 3 intransitive triplets and no transitive triplets. The feed-forward loop, on the other hand, contains 1 transitive triplet and no intransitive triplets. This might also explain the relative rareness of subgraph 10 of Table II, which contain 1 transitive and 2 intransitive triplets, as opposed to 2 transitive and 0 intransitive triplets in subgraphs 9 and 11. Similar transitivity may characterize WWW links [8].

In the neuronal network of $C$. elegans feedback loops and feedback loops with one mutual edge (subgraphs 6 and 10 in Table II) are much less abundant then expected in isotropic geometric networks (Table III). We analyzed two versions of the neuronal network - a network which includes all synaptic connections, and a network which includes only 'strong' connections, where neurons are linked by an edge only if they have 5 or more synapses connecting them.

Interestingly, when one considers subgraphs with no mutual edges (ratios 1,3, 4 in Table III), the full neuronal network of $C$. elegans displays similar subgraph ratios to a highly anisotropic geometric model (with field $p \ll 1$ ). Such a field may represent a directed flow of information from sensory neurons, generally located in the head, to motor neurons. This model cannot however explain the relative abundance of mutual edges, which would not appear in highly biased anisotropic geometric networks.

The subgraph ratios in the neuronal network of strong connections is not consistent with a highly anisotropic geometric model (Table III). It seems that the motifs in this network do not solely stem from geometric constraints, and that additional optimization constraints based on biological functionality are at play $8,11,12,13,14,25]$. Indeed, in transcriptional biological networks, network motifs have been experimentally shown to function as pulse generators 27], asymmetric filters [28], response accelerators [29] and temporal pattern generators [30, 31].

The present scaling results also apply to the class of small-world network models [4]. These models are obtained by rewiring a limited fraction of the edges which leads to a substantial drop in the network diameter but in a small change in the clustering coefficient.

The geometric model can be extended in many ways. One can assign an arbitrary clustering coefficient for each node, in addition to an arbitrary degree, by specifying both the number of edges $k_{i}$ and a neighborhood $R_{i}$ for each node (Eq. 10). This can form a more stringent comparison to networks with broad degree sequences and clustering coefficient sequences [46, 47]. In addition, biases towards mutual edges can be added, in addition to the anisotropy field discussed above.

It would be of interest to extend this study to understand the general relationship between global constraints on a network and its local structure [61, 62, 63, 64].

\section{Acknowledgments}

We thank Ron Milo, Reuven Cohen, Avi Mayo and Tsvi Tlusty for valuable comments. We thank Minerva, HFSP and the Clore center for biological physics for support.

\section{APPENDIX A: OVERLAP INTEGRALS FOR THE HARD-CUBE CONNECTIVITY FUNCTION}

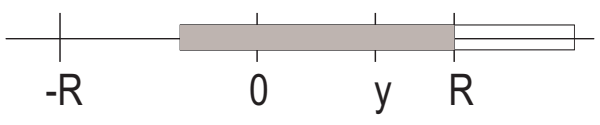

FIG. 5: Overlap area $O(y)=2 R-y$ shaded in gray. All lattice points within this area can connect to both the origin (0) and to $y$.

The intersection integrals reduce to a simple form in the case of the hard-cube connectivity function $F_{c}$. As an example we will consider triangle subgraphs:

$$
\left\langle G_{\Delta}\right\rangle=\frac{N}{6} I_{d}=\frac{N}{6} \iint F(0, y), F(0, z), F(y, z) \overrightarrow{d y} \overrightarrow{d z}
$$

Where $I_{d}$ is the $d$-dimensional overlap integral. In the hard-cube case it is sufficient to calculate the integral in one dimension $I_{1}$. For this connectivity function the axes are separable, and the extension to $d$ dimension is straightforward [57, 58]:

$$
I_{d}=I_{1}^{d}
$$


$I_{1}$ reduces to a one-variable integral :

$$
\begin{array}{r}
I_{1}=\int_{-\infty}^{\infty} \int_{-\infty}^{\infty} F(0, y), F(0, z), F(y, z) d y d z= \\
\left(\frac{k}{(2 R)}\right) \int_{-R}^{R} O(y) d y=\left(\frac{k}{(2 R)}\right)^{3} \int_{-R}^{R}(2 R-|y|) d y= \\
\left(\frac{k}{(2 R)}\right)^{3}\left(3 R^{2}\right)=\frac{3}{2^{3}} k^{3} R^{-1}
\end{array}
$$

Where $O(y)$ is the overlap area, which includes all positions of $z$ which are in the range $R$ of both 0 and $y$ (Fig $5)$.

Extending these results to all directed types of triangles (Table II) and arbitrary dimension yields:

$$
\langle G\rangle=\sigma\left(\frac{3}{2^{g}}\right)^{d} N R^{(n-g-1) d} k^{g}
$$

where $\sigma$ is a symmetry function equal to one over the number of permutations of nodes that lead to an isomorphic subgraph. Eq. A4 contains the scaling of Eq. 9. The prefactors depend on the symmetry function and on the dimension and decrease with $d$. The extension of Eq. A4 to larger subgraphs is :

$$
\langle G\rangle=\sigma\left(\frac{v_{G}}{2^{g}}\right)^{d} N R^{(n-g-1) d} k^{g}
$$

where $v_{G}$ is the factor stemming from the $1 d$ overlap integral, which depends on the non-directed version of $G$. These factors have been calculated in [57] for subgraphs of up to 7 nodes. The prefactors for all non-directed 4-node subgraphs is given in Table II.

\section{APPENDIX B: CALCULATION OF FIELD FACTORS}

Here we describe the method for calculating the field factors for the three-node subgraphs in the $1 d$ anisotropic geometric model. We fix one node at the origin (0). The two remaining nodes are denoted by $y, z$. The formulas for the subgraph occurrences are similar to Eq. 2, but here different integration domains are weighted by different field factors, depending on the orientation of the three nodes. The $y-z$ plane is divided into 6 regions which correspond to the 3 ! linear ordering of the three subgraph nodes. These regions are generally weighted differently. An example for the feedback loop is shown in Fig. 6. For this subgraph symmetries lead to a simplified expression for the field factor $\varphi$ :

$$
\varphi=\frac{1}{2} p q(p+q)=p q
$$

For $n$-node subgraphs one should calculate the $n-1$ dimensional integral over $n$ ! hyper-volumes, corresponding to all linear ordering of the subgraph nodes. For the case

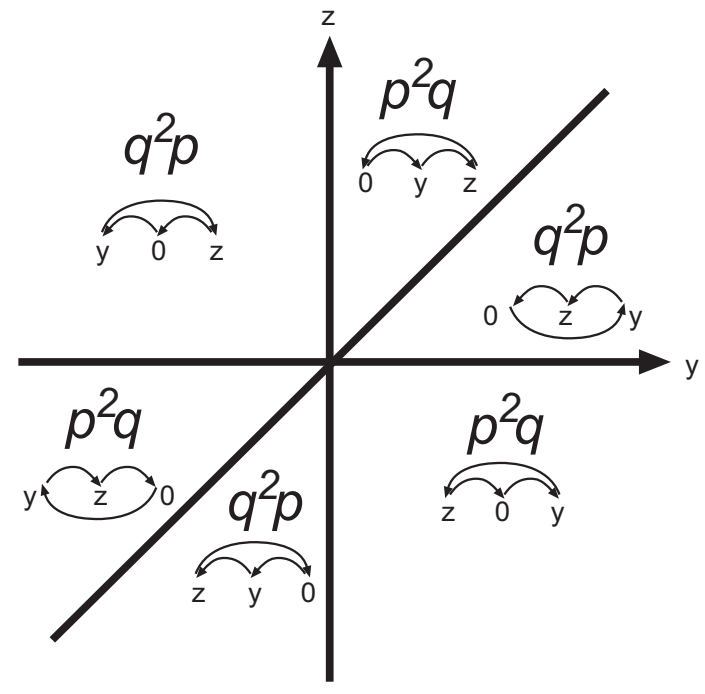

FIG. 6: Orientations used in the calculations of the field factor for the feedback loop in the anisotropic geometric model. The $y-z$ plain is divided into 6 regions of integration, and the weighting factors are shown above the subgraph diagrams.

of the 4-node subgraphs of the invariant ratios presented in Fig. 4(b,c), this calculation results in the following theoretical curves:

$$
r_{b}=\frac{b\left(p^{4}+q^{4}\right)+4(a+c) p q\left(p^{2}+q^{2}\right)+6 b p^{2} q^{2}}{a\left(p^{4}+q^{4}\right)+2 b p q\left(p^{2}+q^{2}\right)+2(a+2 c) p^{2} q^{2}}
$$

where $a=1.1 \cdot 10^{-2}, b=8.8 \cdot 10^{-3}, c=5.4 \cdot 10^{-3}$, and

$$
r_{c}=\frac{1}{2} \frac{h\left(p^{2}+q^{2}\right)+(2 e+2 f+g-h) p q}{e\left(p^{2}+q^{2}\right)+(2 f+g+h) p q}
$$

where $e=2.35 \cdot 10^{-3}, f=1.9 \cdot 10^{-3}, g=3.8 \cdot 10^{-3}, h=$ $1.8 \cdot 10^{-3}$.
[1] S. H. Strogatz, Nature 410, 268-76. (2001).

[2] R. Albert, A.L. Barabasi, Reviews of Modern Physics 74, 47 (2002).

[3] M. E. J. Newman, SIAM Review 45, 167-256 (2003).

[4] D. J. Watts, S. H. Strogatz, Nature 393, 440-442 (1998).
[5] B. M. Waxman, IEEE J. Select Areas. Commun. 6, 1617 (1988).

[6] S. Yook, H. Jeong, and A.-L. Barabasi, Proc. Nat. Acad. Sci 99 13382-13386 (2002).

[7] G. Bianconi, G. Caldarelli, A. Capocci, 
cond-mat/0408349 (2004).

[8] R. Milo, S. Itzkovitz, N. Kashtan, et al., Science 303, 5663 (2004).

[9] J. R. Banavar, A. Maritan, A. Rinaldo, Nature 13;399(6732):130-2 (1999).

[10] P. Sen, S. Dasgupta, A. Chatterjee, P. A. Sreeram, G. Mukherjee, and S. S. Manna Phys. Rev. E 67, 036106 (2003).

[11] J. G. White, E. Southgate, J. N. Thomson, and S. Brenner Phil. Trans. R. Soc. London, B 314, 1-340 (1986).

[12] T. C. Ferree, S.R. Lockery, J. Computat. Neurosci. 6:263277 (1999).

[13] O. Hobert, J. Neurobiol. 54(1):203-23 (2003).

[14] N. Kashtan, S. Itzkovitz, R. Milo, U. Alon, Phys. Rev. E in press (2004).

[15] C. Cherniak, Z. Mokhtarzada, R. Rodriguez-Esteban, K. Changizi, Proc. Nat. Acad. Sci 101, 1081-1086 (2004).

[16] M. Kaiser and C.C. Hilgetag, Phys. Rev. E 69, 036103 (2004).

[17] S. Wasserman, K. Faust, Social Network Analysis (Cambridge University Press, cambridge 1994).

[18] A. L. Barabasi, and R. Albert, Science,286, 509-512 (1999).

[19] J. Eckmann, E. Moses, PNAS 99, 5825-5829 (2002).

[20] J. A. Davis, Perspectives on Social Network Research, edited by Paul W. Holland and Samuel Leinhardt. New York: Academic Press, pp 51-62 (1979).

[21] S. Maslov, Y-C. Zhang, Phys. Rev. Lett. 87, 248701 (2001).

[22] J. Ihmels, G. Friedlander, S. Bergmann, O. Sarig, Y. Ziv and N. Barkai, Nature Genetics 31/4, 370-377 (2002).

[23] R. N. Mantegna, Eur. Phys. J. B 11, 193 (1999).

[24] S. Shen-Orr, R. Milo, S. Mangan, et al.,Nat Genet 31, 64 (2002).

[25] R. Milo, S. Shen-Orr, S. Itzkovitz, et al., Science 298, 824 (2002).

[26] U. Alon, Science, 301:1866-1867 (2003).

[27] S. Mangan and U. Alon, Proc Natl Acad Sci U S A 100, 11980 (2003).

[28] S. Mangan, A. Zaslaver, and U. Alon, JMB 334, 197 (2003).

[29] N. Rosenfeld, M. B. Elowitz, and U. Alon, J Mol Biol 323, 785 (2002).

[30] M. Ronen, R. Rosenberg, B. I. Shraiman, et al., Proc Natl Acad Sci U S A 99, 10555 (2002).

[31] A. Zaslaver, A. Mayo, R. Rosenberg, et al., Nat Genet 36, 486 - 491 (2004).

[32] G. Lahav, N. Rosenfeld, A. Sigal, et al., Nat Genet 36:147-150 (2004).

[33] Y. Artzy-Randrup, S.J. Fleishman, N. Ben-Tal, L. Stone, Science 305: 1106c-1107c, (2004).

[34] R. Milo, S. Itzkovitz, N. Kashtan, R. Levitt, U. Alon, Science 305:5687, (2004).

[35] C. Herrmann, M. Barthelemy, P. Provero, Phys Rev E 68, 026128 (2003).

[36] M. Barthelemy, europhysics letters 63-6(2003).

[37] M. T. Gastner, M. E. J. Newman, cond-mat/0407680 (2004).

[38] J. Dall, M. Christensen, Phys Rev E 66, 016121 (2002).

[39] A. F. Rozenfeld, R. Cohen, D. ben-Avraham, and S.
Havlin, Phys. Rev. Lett. 89, 218701 (2002).

[40] P. Erdős \& A. Rényi, Publicationes Mathematicae 6, 290297 (1959).

[41] P. Erdős \& A. Rényi, Publications of the Mathematical Institute of the Hungarian Academy of Sciences 5, 17-61 (1960).

[42] P. Erdős \& A. Rényi, Acta Mathematica Scientia Hungary 12, 261-267 (1961).

[43] B. Bollobas, Random Graphs, Academic Press, New York (1985).

[44] S. Itzkovitz, R. Milo, N. Kastan, et al., Phys Rev E 68, 026127 (2003).

[45] Z. Burda, J. Jurkiewicz, A. Krzywicki, Phys. Rev. E69 026106 (2004).

[46] E. Ravasz, A. L. Somera, D. A. Mongru, et al., Science 297, 1551 (2002).

[47] A. Vazquez, R. Dobrin, D. Sergi, J. P. Eckmann, Z. N. Oltvai, A. L. Barabasi, cond-mat/0408431 (2004).

[48] A. L. Barabasi \& R. Albert, Science 286, 509-12 (1999).

[49] S. Redner, European Phys. J. B 4, 131 (1998).

[50] M. Faloutsos , P. Faloutsos , C. Faloutsos, Comp. Comm. Rev. 29, 251-262 (1999).

[51] B. A. Huberman, L. A. Adamic, Nature 401, 131 (Sep 1999).

[52] R. Cohen, K. Erez, D. ben-Avraham, S. Havlin, Phys. Rev. Lett. 85, 4626 (2000).

[53] S. N. Dorogovtsev, A. N. Samukhin, Phys. Rev. E 67, 037103 (2003).

[54] C. R. Myers, Phys. Rev. E 68, 046116 (2003).

[55] S. Valverde, R. V. Sole, cond-mat/0307278 (2003).

[56] N. Kashtan, S. Itzkovitz, R. Milo, U. Alon, Bioinformatics $22 ; 20(11): 1746-58$ (2004).

[57] W. G. Hoover, A. G. De-Rocco, J. Chem. Phys., 36:31413162 (1962).

[58] A. Drory, I. Balberg, U. Alon, B. Berkowitz, Phys. Rev. A 43, 6604-6612 (1991).

[59] P. Holland, S. Leinhardt, D. Heise, Eds., in Sociological Methodology (Jossey-Bass, San Francisco, 1975), pp. 145.

[60] D. Cartwright, F. Harary, Psychol. Rev. 63, 277 (1956).

[61] J. Berg J, M. Lassig, Phys. Rev. Lett. 89 (22),228701 (2002).

[62] G. Palla, I. Derenyi, I. Farkas, T. Vicsek, Phys. Rev. E 69, 046117 (2004).

[63] E. A. Variano, J. H. McCoy, H. Lipson, Phys. Rev. Lett. 92(18), 188701 (2004).

[64] J. Park, M.E.J. Newman, cond-mat/0405566 (2004).

[65] The degree $k$ is the sum of random independent poissonian variables $k(r)$ which represent the number of links to nodes within a shell at distance $r$.

[66] This statement relate to a random ensemble of geometric networks which does not preserve the degree sequences of the real network, only the mean connectivity and clustering coefficient (the Poissonian geometric model). In a more stringent random ensemble which preserves the degree sequences, the subgraph ratios change and depend on different moments of the degree sequences (section IID, [44]). 